\title{
Non-anesthetist-administered moderate sedation with midazolam and fentanyl for outpatient MRI-aided hybrid intracavitary and interstitial brachytherapy in cervix cancer: a single-institution experience
}

\author{
Kiattisa Sommat, MBBS, MRCP, FRCR, MCl', Jeannie Yi Xin Lin, MScl, Melvin Ming Long Chew, BSc', Chiat Sian Loh, BN', \\ Jorene Siew Kee Liew, BN', Yong Wee Foo, MSc', Jin Wei Kwek, MBBS, FRCR², Tiffany Hennedige, MBBS, FRCR, MMed, \\ $\mathrm{MCl}^{2}$, June Pheck Suan Goh, MBBS, FRCA ${ }^{3}$, Tong Khee Tan, MBBS, FRCA ${ }^{3}$ \\ 'Division of Radiation Oncology, National Cancer Centre Singapore, Singapore, 2Division of Oncologic Imaging, National Cancer Centre \\ Singapore, Singapore, ${ }^{3}$ Department of Anesthesiology, Singapore General Hospital, Singapore
}

\begin{abstract}
Purpose: The aim of the study was to determine the feasibility of interstitial brachytherapy under non-anesthetist-administered moderate sedation, to identify factors influencing the insertion, and the total procedural time.

Material and methods: A total of 47 insertions with hybrid intracavitary and interstitial applicators were performed in 23 patients from March 2017 to March 2020. Moderate sedation was achieved with intravenous midazolam and fentanyl administered by non-anesthetist. Insertion time and procedural time was recorded. Univariate and multivariate analysis were performed to evaluate the impact of different factors on insertion and procedural time.

Results: A total of 238 needles (range, 2-8 per insertion) were implanted, with an average insertion depth of $30 \mathrm{~mm}$ (range, 20-40 mm). The mean doses for midazolam and fentanyl were $3 \mathrm{mg}$ (standard deviation [SD] = 1) and $53.3 \mathrm{mcg}$ $(\mathrm{SD}=23.9)$ per insertion, respectively. The median insertion time was 30 minutes (interquartile range $[\mathrm{IQR}]=22-40)$, and the median total procedural time was 4.3 hours $(\mathrm{IQR}=3.6-5.2)$. First time insertion, insertions performed before 2019, and higher midazolam dose were associated with significantly longer insertion time, whereas longer insertion time, MRIbased planning, and insertions performed before 2019 were associated with significantly longer total procedural time.

Conclusions: Outpatient interstitial brachytherapy with non-anesthetist-administered sedation is achievable and well-tolerated. This method may significantly lessen the burden on hospital resources and has the potential to be cost-effective.

Key words: interstitial brachytherapy, sedation, MRI-aided.

\section{Purpose}

Interstitial brachytherapy is indicated in the treatment of cervix cancer when the tumor extension cannot be adequately covered by intracavitary brachytherapy [1-4]. Sedation and pain management are required as interstitial brachytherapy is an inherently invasive, unpleasant, and painful procedure [5]. Various anesthesia approaches have been used, from sedation and neuraxial anesthesia to general anesthesia, depending on institutional logistics or cost constraints [5-7]. Although an anesthetist-led service is often preferred, it is a scarce and expensive resource in many institutions. At the time

of writing, many brachytherapy practitioners across the world are facing limited access to anesthetists or hospital beds due to the ongoing COVID-19 pandemic [8,9]. Various groups have published recommendations regarding the use of anesthesia for gynecological brachytherapy in response to these challenging circumstances related to COVID-19. In general, moderate sedation in an outpatient setting with omission of general anesthesia is preferred. As such, the use of safe and effective sedation for interstitial brachytherapy without an involvement of anesthetist is most desirable to ensure timely completion of brachytherapy despite limited resources.
\end{abstract}

\footnotetext{
Address for correspondence: Kiattisa Sommat, Division of Radiation Oncology, National Cancer Centre 
A sedation continuum has been described by the American Society of Anesthesiologists (ASA), ranging from mild sedation to general anesthesia. Moderate sedation with analgesia (formerly known as "conscious sedation") is the depression of consciousness, during which the patients respond purposefully to verbal commands or with light tactile stimulation. Moderate sedation/analgesia with midazolam and/or fentanyl was prospectively reported to be safe and therefore, the preferred drug choice for not supervised by an anesthetist cases [10-12]. While moderate sedation has been widely performed by brachytherapists for intracavitary brachytherapy, there is not much published reports on the use of moderate sedation for interstitial brachytherapy [13]. Practice guidelines for non-anesthetists providing sedation have been put forth by various professional bodies, including the ASA and the College of Anesthesiologists, Academy of Medicine Singapore [14, 15]. The potential advantages of non-anesthetists-administered moderate sedation (formerly known as "conscious sedation") are multifold, and include lower cost, shorter post-procedural recovery, and wider availability. Furthermore, technological advances in brachytherapy applicator design allow plastic interstitial needles to be placed in combination with intracavitary applicators. These modern hybrid applicators enable quick and relatively easy applicator assembly and insertion, while reducing the need for free-hand needle placement, resulting in shorter procedural time and increased patients' comfort [16-19].

In January 2017, we established interstitial brachytherapy service in our institution to treat patients with cervical cancer. The first few insertions were performed under anesthetist-administered deep sedation or general anesthesia (GA) in an operating theatre. However, anesthetist covering was neither readily nor regularly available, as the operating theatre was a shared resource with other surgical specialties. Faced with limited access to anesthetist covering and after a discussion with our anesthesia colleagues, we started to perform interstitial brachytherapy insertions under non-anesthetist-administered moderate sedation in the operating theatre and later on, in a radiation oncology facility. Currently, published reports on the use of moderate sedation for interstitial brachytherapy are rare. Therefore, the aim of our study was to assess the feasibility of moderate sedation for interstitial brachytherapy, using hybrid intracavitary and interstitial applicators in the outpatient setting, and to identify factors influencing the insertion and procedural time.

\section{Material and methods}

This was a retrospective review of patients, with biopsy-proven cervical cancer who underwent interstitial brachytherapy using hybrid intracavitary and interstitial applicators from March 2017 to March 2020. During this period, a total of twenty-three consecutive patients with non-metastatic cervical cancer and the ASA physical status I-II underwent forty-seven insertions of interstitial brachytherapy in department of radiation oncology (DRO) of the National Cancer Centre Singapore. All patients were staged according to the International Federa- tion of Gynecology and Obstetrics (FIGO) staging system [20]. All patients signed an informed consent form allowing their clinical data to be collected and used for the purpose of research. Data were prospectively documented, and retrospectively collected and analyzed.

All patients were treated with external beam radiotherapy (EBRT), between 45 Gy and $50.4 \mathrm{~Gy}$, in $1.8 \mathrm{~Gy}$ per fraction. Chemotherapy consisted of weekly cisplatin of $40 \mathrm{mg} / \mathrm{m}^{2}$ up to six cycles was given concurrently with EBRT. Brachytherapy was commenced either within the final week or within 1 week of finishing EBRT. No chemotherapy or EBRT were allowed on the days of brachytherapy.

\section{Patients' selection and pre-sedation assessment}

One week before the first session of brachytherapy, all patients underwent magnetic resonance imaging (MRI) of pelvis without applicator in situ to evaluate the topography of residual tumor and to identify the need for interstitial needles based on the predicted isodose distribution of intracavitary brachytherapy alone in order to increase the likelihood of optimal needle placement at the actual time of brachytherapy application. The number, position, and depths of the required needles were pre-planned by a radiation oncologist, with inputs from a radiologist and brachytherapy physicist. MRI T2-weighted images were used, taking into account an offset of $5 \mathrm{~mm}$ from the tip of the needle to the first dwell position.

A dedicated interstitial brachytherapy insertion suite was established in DRO in 2019. Patient-related procedures involved five different rooms, such as insertion suite, computed tomography (CT) simulator, MRI scanner, observation room, and brachytherapy treatment room, all located in the same building and on the same floor. Figure 1 shows the simplified floor plan of the interstitial brachytherapy facility at the DRO. Before 2019, all interstitial brachytherapy insertions were performed in an operating theatre in the same building, but on a different floor. In every case, the same radiation oncologist performed the interstitial brachytherapy procedure from pre-planning to removal of applicators. The types of hybrid applicators in our department were tandem and ring-based: interstitial ring $\mathrm{CT} / \mathrm{MR}$ applicator, and

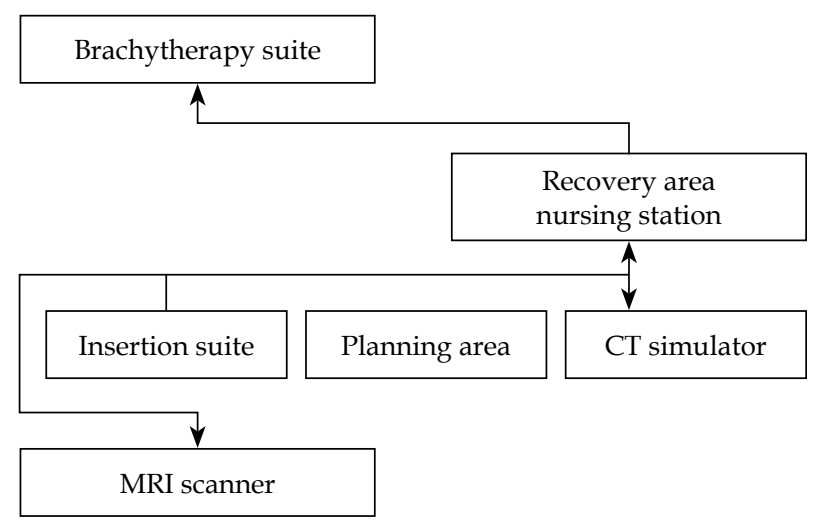

Fig. 1. Simplified floor plan of the interstitial brachytherapy facility at the National Cancer Centre Singapore 
tandem and ovoids-based: Venezia advanced gynecological applicator and Utrecht interstitial CT/MR applicator. The choice of applicator type was determined by the radiation oncologist, based on applicator availability, patient's anatomy, and tumor topography.

All patients were assessed by the same radiation oncologist before brachytherapy, with a written consent for procedure and sedation obtained. Pre-brachytherapy evaluation included an assignment of ASA physiologic status classification [21] based on patient's medical history, physical examination, and review of pre-brachytherapy laboratory tests. Anesthetist referral and management are mandatory for severely compromised or medically unstable patients (e.g., ASA status III or IV, anticipated difficult airway, or severe congestive heart failure). If there was a doubt surrounding the safety or feasibility, such as anxious or phobic patients to undergo non-anesthetist-administered sedation, an anesthetic review was obtained.

\section{Pre-brachytherapy preparation}

On the evening before brachytherapy, the patients took oral bowel preparation with $45 \mathrm{ml}$ of fleet-phospho-soda and were fasting for at least 6 hours. Upon arrival at our department, the patients were assessed again by a dedicated seditionist. A seditionist refers to the person who administers the sedation and monitors the patient during this process until full recovery. The seditionist is distinct from the proceduralist and does not perform any other tasks, i.e., assisting with the procedure. At the DRO, all brachytherapy practitioners, medical officers, and specialist trainees are qualified seditionist by an accredited training. We recognize that other allied health staffs, such as nurses, may fulfill this role depending on institutional practices. All staff involved in brachytherapy procedures are at minimum competent, with basic resuscitation skills.

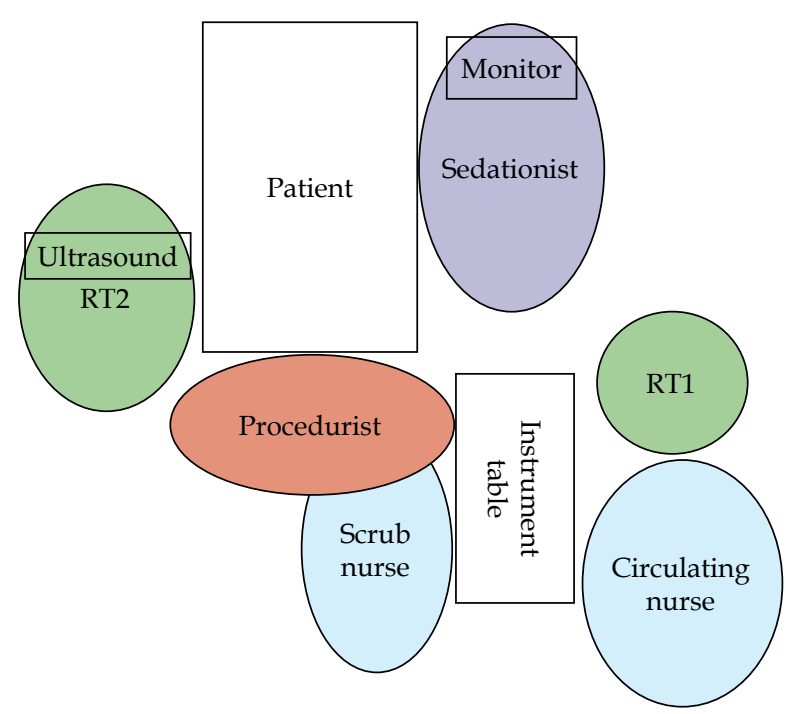

Fig. 2. Overview of the insertion suite layout. RT1 - radiotherapist 1, RT2 - radiotherapist 2

\section{Applicator insertion under sedation}

Shortly before brachytherapy, a quick huddle was led by the procedurist in the presence of immediate members of the brachytherapy team, including seditionist, scrub nurse, circulating nurse, physicist, and two radiation therapists involved in brachytherapy. Radiotherapist 1 (RT1) was in charge of time out, verification, and documentation of applicator and needles used, whereas radiotherapist 2 (RT2) was responsible for operating the ultrasound machine. An overview of the insertion suite layout is shown in Figure 2. The patients were placed in lithotomy position and following surgical time-out, an initial dose of intravenous midazolam and fentanyl was given by the seditionist under the direction of the procedurist. Starting dose of midazolam and fentanyl was 1-2 $\mathrm{mg}$ and 10-20 mcg, respectively, with repeated dosing every 2-5 minutes as needed, based on an assessment of pain in communicative patients throughout the insertion, to a maximum total dose of $5 \mathrm{mg}$ for midazolam and $100 \mathrm{mcg}$ for fentanyl. We used a Ramsay sedation scale to grade the level of sedation. Vital signs were monitored before, throughout, and every 5 minutes after the applicator insertion until discharge. Supplemental oxygen could be administered if oxygen saturation decreased to $90 \%$ or less. Standard resuscitation equipment and reversal drugs for fentanyl (naloxone) and midazolam (flumazenil) were available during all procedures.

A Foley catheter was inserted and the uterus was sounded under ultrasound guidance to ascertain the required length of the tandem. Local vaginal anesthesia with $2 \%$ lidocaine gel was administered. Plastic interstitial needles with blunt tips were inserted to the pre-planned depth using an insertion tool, following the placement of tandem and ring/ovoids/semi-lunar ovoids. The insertion depth was defined as the length of the needle from its tip to the surface of the ring or ovoids. The applicators were immobilized with gauze packing, which also aided in sparing of the rectum and bladder. The patients were then transferred to CT simulator and adjustments of needle depths were made if required. If MRI slots were available, the patients would then be transferred to department of oncologic imaging for MRI scan after CT scan.

\section{Applicator removal and discharge}

During the time of contouring and dosimetric planning, the patients were placed in an observation room next to nursing station for monitoring. We applied volume-based prescription to high-risk clinical target volume (HR-CTV). Our planning aim was to deliver a HR-CTV $\mathrm{D}_{90}$ (dose received by $\geq 90 \%$ of volume) $E Q D_{2}$ (equivalent dose at 2 Gy per fraction) of at least $87 \mathrm{~Gy}$ [22]. After the treatment plan was approved, the patients were move to the brachytherapy treatment room. We used MicroSelectron HDR (Nucletron, Sweden) for the treatment, and ${ }^{192} \mathrm{Ir}$ was applied as the treatment source. The applicator and needles were then removed, and vaginal packing was inserted and pressure applied. Patient could receive top up of IV fentanyl during applicator removal. The cervix and 
vagina were examined for bleeding and the patients were monitored for at least 30 minutes after the removal of applicators. All the patients were assessed by a physician before their discharge. We used post-anesthetic discharge scoring system (PADSS) score of 9 or higher as criteria for discharge. All the patients musted be accompanied home by a responsible adult. Insertion time (from time out to end of vaginal packing) and total procedural time (from time out to applicator removal) were recorded.

\section{Statistical analysis}

Analyses were performed to examine the associations between insertion time (minutes) or total procedural time (hours), and factors, including applicator type (interstitial ring vs. Utrecht/Venezia), number of insertions (first vs. second and third), number of needles ( $\leq 5 \mathrm{vs} .>5$ ), period of insertions (2017 to 2018 vs. 2019 to 2020), MRI-based planning (no vs. yes), age (continuous), fentanyl dose (continuous), and midazolam dose (continuous). Student $t$-tests were performed for bivariate analysis, whereas continuous variables were correlated to insertion time

Table 1. Patients' and tumors' characteristics $(n=23)$

\section{Variable}

\begin{tabular}{|c|c|}
\hline Age (year), mean (range) & $55(27-77)$ \\
\hline \multicolumn{2}{|l|}{ Histology, n (\%) } \\
\hline Squamous cell carcinoma & 18 (78.3) \\
\hline Adenocarcinoma & $3(13)$ \\
\hline Other & $2(8.7)$ \\
\hline \multicolumn{2}{|l|}{ FIGO stage, $n(\%)$} \\
\hline IB2 & $1(4.3)$ \\
\hline$\| B$ & $9(39.2)$ \\
\hline IIIB & $10(43.5)$ \\
\hline IIIC1 & $1(4.3)$ \\
\hline IIIC2 & $2(8.7)$ \\
\hline \multicolumn{2}{|l|}{ ASA status, $n(\%)$} \\
\hline 1 & $17(73.9)$ \\
\hline$\|$ & $6(26.1)$ \\
\hline \multicolumn{2}{|l|}{ Chemotherapy use, $n$ (\%) } \\
\hline No & $1(4.3)$ \\
\hline Yes & $22(95.7)$ \\
\hline \multicolumn{2}{|l|}{ EBRT technique, $n(\%)$} \\
\hline 3D-CRT & 17 (73.9) \\
\hline VMAT & $6(26.1)$ \\
\hline
\end{tabular}

FIGO - International Federation of Gynecology and Obstetrics, ASA - American Society of Anesthesiologists, EBRT - external beam radiotherapy, 3D-CRT - 3-dimenstional conformal radiotherapy, VMAT - volumetric-modulated arc therapy or total procedural time using Pearson correlation. All variables with $p$-value less than 0.05 were considered for inclusion in a multiple linear regression. All statistical analysis was carried out using STATA software, version 14.1 (StataCorp LP, Texas, USA).

Table 2. Interstitial brachytherapy applications' characteristics of 47 insertions

\begin{tabular}{|c|c|}
\hline Variable & \\
\hline \multicolumn{2}{|l|}{ Applicator type } \\
\hline Interstitial ring & $10(21.3)$ \\
\hline Utrecht & $19(40.4)$ \\
\hline Venezia & $18(38.3)$ \\
\hline \multicolumn{2}{|l|}{ No. of insertions } \\
\hline First & $23(48.9)$ \\
\hline Second & $18(38.3)$ \\
\hline Third & $6(12.8)$ \\
\hline \multicolumn{2}{|l|}{ Period of insertions } \\
\hline 2017 to 2018 & $21(44.7)$ \\
\hline 2019 to 2020 & $26(55.3)$ \\
\hline No. of needles, mean (range) & $5(2-8)$ \\
\hline Depth of needles (cm), mean (range) & $3(2-4)$ \\
\hline Midazolam dose (mg), mean (range) & $3(0.5-5)$ \\
\hline Fentanyl dose (mcg), mean (range) & $53.3(10-100)$ \\
\hline \multicolumn{2}{|l|}{ Insertion time (min) } \\
\hline Mean & 32.4 \\
\hline Median & 30.0 \\
\hline $\mathrm{IQR}$ & $22-40$ \\
\hline Range & $12-85$ \\
\hline \multicolumn{2}{|l|}{ Total procedural time (hours) } \\
\hline Mean & 4.5 \\
\hline Median & 4.3 \\
\hline $\mathrm{IQR}$ & $3.6-5.2$ \\
\hline Range & 2.9-7.1 \\
\hline Fractional HR-CTV D 90 (Gy), median (range) & $8(6.2-9.9)$ \\
\hline Fractional IR-CTV D 90 (Gy), median (range) & $5.9(3.1-7.3)$ \\
\hline Fractional rectum $\mathrm{D}_{2 c c}(\mathrm{~Gy})$, median (range) & $4.7(1.3-6.3)$ \\
\hline Fractional bladder $\mathrm{D}_{2 c c}(\mathrm{~Gy})$, median (range) & $5.6(3.3-7.1)$ \\
\hline Fractional sigmoid $D_{2 c c}(G y)$, median (range) & $4.2(2.3-5.8)$ \\
\hline Total EQD 2 HR-CTV $D_{90}(G y)$, median (range) & $85.7(80-92.9)$ \\
\hline Total EQD 2 IR-CTV D ${ }_{90}(G y)$, median (range) & $73.4(66.4-75.7)$ \\
\hline Total $E Q D_{2}$ rectum $D_{2 c c}(G y)$, median (range) & $71.8(63.7-75.9)$ \\
\hline Total EQD 2 bladder $\mathrm{D}_{2 \mathrm{cc}}(\mathrm{Gy})$, median (range) & $82.5(54.8-88.4)$ \\
\hline Total EQD ${ }_{2}$ sigmoid $\mathrm{D}_{2 c c}(\mathrm{~Gy})$, median (range) & $69.8(58.9-75.4)$ \\
\hline
\end{tabular}

$I Q R$ - interquartile range, $H R$ - high-risk, IR - intermediate-risk, CTV - clinical target volume, $D_{90}$ - dose received by $\geq 90 \%$ of volume, $D_{100}$-dose received by $100 \%$ of volume, $D_{2 c c}$-dose to the most irradiated $2 c c, E Q D_{2}$ - biologically equivalent dose in 2 Gy fractions 


\section{Results}

Patients and treatment characteristics are presented in Table 1. The patients' mean age at diagnosis was fifty-five years, with a range of 27-77 years. Most of the patients had squamous cell carcinoma ( $n=18,78.2 \%)$. Ten patients $(43.5 \%)$ had pelvic side wall involvement at diagnosis. The majority of patients $(n=17,73.9 \%)$ received 3-dimenstional conformal radiotherapy (3D-CRT) and the remaining 6 patients $(26.1 \%)$ received volumetric-modulated arc therapy (VMAT).

A total of forty-seven hybrid intracavitary and interstitial brachytherapy insertions were performed in twenty-three patients (Table 2). As for the applicator, interstitial ring was used in 10 insertions, Utrecht applicator was used in 19 insertions, and Venezia applicator was used in 18 insertions. Overall, 238 needles (range, 2-8 per insertion) were implanted, with an average insertion depth of $30 \mathrm{~mm}$ (range, 20-40 $\mathrm{mm}$ ). The mean doses for midazolam and fentanyl were $3 \mathrm{mg}$ (standard deviation [SD] = 1) and $53.3 \mathrm{mcg}(\mathrm{SD}=23.9)$ per insertion, respectively. MRIbased planning was carried out in eleven $(23.4 \%)$ out of forty-seven insertions, whereas CT-based planning was performed in the remaining thirty-six $(76.6 \%)$ patients. The median insertion time was 30 minutes (interquartile range $[\mathrm{IQR}]=22-40$ ), and the median total procedural time was 4.3 hours (IQR $=3.6-5.2)$. The median fractional $\mathrm{D}_{90}$ of HR-CTV was 8 Gy (range, 6.2-9.9 Gy), and the median total $\mathrm{D}_{90} \mathrm{EQD}_{2}$ of HR-CTV and intermediate-risk clinical target volume (IR-CTV) was 85.7 Gy (range, 80-92.9 Gy) and 73.4 Gy (range, 66.4-75.7 Gy), respectively. The median total $\mathrm{D}_{2 \mathrm{cc}}$ (dose to the most irradiated 2cc of volume) $\mathrm{EQD}_{2}$ of rectum, bladder, and sigmoid colon was $71.8 \mathrm{~Gy}, 82.5 \mathrm{~Gy}$, and $69.8 \mathrm{~Gy}$, respectively.

The results of the univariate analyses are summarized in Tables 3 and 4. In the univariate analysis, we observed that first attempt insertion, insertions performed in the earlier period between 2017 to 2018, and insertions requiring MRI-based planning were associated with longer insertion time and total procedural time. Higher midazolam dose was also associated with longer insertion time. A shorter insertion time was found to be associated with a shorter total procedural time. No statistically significant difference was observed between Venezia, Utrecht, and interstitial ring applicator.

Table 3. Univariate analysis of factors influencing insertion and total procedural time

\begin{tabular}{|c|c|c|c|c|c|c|c|}
\hline \multirow[t]{2}{*}{ Sub-group } & \multirow[t]{2}{*}{ No. } & \multicolumn{3}{|c|}{ Insertion time (minutes) } & \multicolumn{3}{|c|}{ Total procedural time (hours) } \\
\hline & & Mean & SD & $p$-value & Mean & SD & $p$-value \\
\hline \multicolumn{8}{|l|}{ Period of insertions } \\
\hline 2017 to 2018 & 21 & 38.1 & 11.3 & & 5.4 & 1.0 & \\
\hline 2019 to 2020 & 26 & 27.8 & 16.0 & $0.017^{\star}$ & 3.8 & 0.7 & $<0.003^{*}$ \\
\hline \multicolumn{8}{|l|}{ Applicator type } \\
\hline Interstitial ring & 10 & 34.1 & 7.3 & & 5.0 & 1.2 & \\
\hline Utrecht or Venezia & 37 & 31.9 & 16.4 & 0.69 & 4.4 & 1.1 & 0.11 \\
\hline \multicolumn{8}{|l|}{ No. of insertions } \\
\hline First & 23 & 39.8 & 16.3 & & 5.0 & 1.3 & \\
\hline Second or third & 24 & 25.3 & 9.1 & $<0.003^{*}$ & 4.0 & 0.9 & $0.005^{*}$ \\
\hline \multicolumn{8}{|l|}{ No. of needles } \\
\hline$\leq 5$ & 28 & 32.6 & 18.4 & & 4.3 & 1.2 & \\
\hline$>5$ & 19 & 32.1 & 7.7 & 0.911 & 4.8 & 1.1 & 0.12 \\
\hline \multicolumn{8}{|l|}{ MRI-based planning } \\
\hline No & 36 & 29.8 & 15.5 & & 4.1 & 0.9 & \\
\hline Yes & 11 & 40.9 & 8.9 & $0.029^{*}$ & 5.9 & 1.0 & $<0.003^{*}$ \\
\hline
\end{tabular}

${ }^{*} p<0.05$ and included in multivariate model building, MRI - magnetic resonance imaging

Table 4. Correlation analysis of insertion and total procedural time according to continuous variables Insertion time (minutes)

Total procedural time (hours)

\begin{tabular}{lcccc} 
& Correlation coefficient, $r$ & $p$-value & Correlation coefficient, $r$ & $p$-value \\
\hline Age (years) & 0.089 & 0.55 & 0.064 & 0.667 \\
\hline Fentanyl dose $(\mathrm{mcg})$ & 0.226 & 0.127 & -0.169 & 0.257 \\
\hline Midazolam dose $(\mathrm{mg})$ & 0.304 & $0.038^{\star}$ & 0.12 & 0.421 \\
\hline Insertion time $(\mathrm{min})$ & N.A. & N.A. & 0.697 & $<0.003^{\star}$
\end{tabular}

${ }^{*} p<0.05$ and included in multivariate model building, N.A. - not applied 
In the multivariate analysis, higher midazolam dose, first attempt insertion, and insertions performed before 2019 were associated with significantly prolonged insertion time, whereas longer insertion time, MRI-based planning, and insertions performed before 2019 were associated with significantly prolonged total procedural time. The average time needed for the first insertion was 39.8 minutes and was decreased by 14.8 minutes (95\% CI: -23.4 to $-6.2 \mathrm{~min}, p=0.001$ ) in subsequent insertions. The average insertion time was also decreased by $10.5 \mathrm{~min}-$ utes from earlier period of 2017-2018 to later period of 2019-2020.

The average total procedural time was decreased by 0.9 hours after 2019 (95\% CI: $-1.31 \%$ to $-0.41 \%, p<0.003$ ). As expected, MRI-based planning was associated with an increase of 0.82 hours to the total procedural time (95\% CI: $0.25 \%$ to $1.38 \%, p=0.005$ ) (Table 5).

All the patients underwent the procedure successful$1 y$, and none of the 47 insertions were aborted. Moreover, no patient was unable or unwilling to complete her prescribed brachytherapy sessions due to pain or discomfort. There were no obvious complications, such as uterine perforation, during the insertions. There were no adverse events attributed to the moderate sedation. At the time of discharge, all the patients reported pain score of 0-1 and achieved PADSS score of 10 . No case of severe bleeding or infections, which required transfusion or hospitalization was observed.

\section{Discussion}

To our knowledge, this is one of the largest series in the literature reporting the use of non-anesthetist-administered moderate sedation for hybrid intracavitary and interstitial brachytherapy in patients with cervical cancer. The results from our study demonstrated that interstitial brachytherapy can be successfully introduced into a radiotherapy department in the outpatient setting. Although the placement of interstitial needles posed minimal procedural risk, the perceived requirement for anesthesia often complicates scheduling and delivery. Moderate sedation does not require highly specialized and expensive equipment, and is associated with very low complication rates [23]. Furthermore, moderate sedation can be administered by specially trained personnel and thus, it would not require an anesthetist to attend $[15,24]$.
Benrath et al. conducted a review of 1,622 anesthetic procedures used for brachytherapy [7]. Complications of GA was documented in 35\% of patients and dominated by cardiovascular incidents, such as hypotension and bradycardia. For regional anesthesia, they reported that hypotension occurred in $10 \%$, bradycardia in $10 \%$, and technical complications (multiple puncture attempts, puncture of the dura during epidural placement, bloody puncture, etc.) in $4 \%$. We have made great efforts to develop high standards of safety, in terms of training and accreditation, to ensure competency and proficiency in the skills required for sedation. None of the patients in this study experienced any complications related to sedation during and after the procedure. Doubts about the feasibility of moderate sedation for hybrid intracavitary and interstitial brachytherapy should be tempered by the fact that all the 47 insertions were successfully completed and well tolerated.

So far, in existing publications on interstitial brachytherapy with modern hybrid applicators involving deep sedation, general, or spinal anesthesia, the procedures required the presence of anesthetist. Leong et al. reported their experience of 35 insertions using Utrecht applicators performed under moderate to deep sedation administered by anesthetist, combining with paracervical block and local anesthesia [25]. A recent study by Walter et al. presented their initial results of Venezia applicator in 10 patients, where insertions were performed under general or spinal anesthesia [18]. Another study reported insertions using Vienna ring applicator under spinal anesthesia in 22 patients [26].

In this study, we found that the mean applicator insertion time and total procedural time was $32.4 \pm 14.9$ minutes and $4.5 \pm 1.2$ hours, respectively, which are comparable to results from other groups. Similar study by Leong and colleagues reported mean insertion time of 39 minutes and mean time to discharge of 4.1 hours [25]. Liu et al. evaluated a technique of interstitial brachytherapy in 28 patients using applicator combining uterine tandem and metal needles, where the needles were inserted free-hand under real-time CT guidance [27]. All patients underwent spinal anesthesia for interstitial brachytherapy, and mean insertion time was 41.2 minutes. In comparison to intracavitary brachytherapy, Mayadev et al. described a mean insertion time of 22.6 minutes in 217 insertions, with moderate sedation administered by nurses under the supervision of brachytherapist [28].

Table 5. Multivariate analysis of factors influencing insertion and total procedural time

\begin{tabular}{lcccccc} 
& \multicolumn{3}{c}{ Insertion time (minutes) } & \multicolumn{2}{c}{ Total procedural time (hours) } \\
\cline { 2 - 8 } & $\beta^{*}$ & $95 \% \mathrm{Cl}$ & $p$-value & $\beta^{*}$ & $95 \% \mathrm{Cl}$ & $p$-value \\
\hline Midazolam dose (mg) & 4.15 & $0.23-8.1$ & 0.038 & & - & \\
\hline Period of insertions (2017-2018 vs. 2019-2020) & -14.1 & -24.00 to -4.20 & 0.006 & -0.86 & -1.31 to -0.41 & $<0.003$ \\
\hline No. of insertions (first vs. second or third) & -13.0 & -21.42 to -4.62 & 0.003 & 0.07 & -0.36 to 0.50 & 0.736 \\
\hline MRI-based planning (no vs. yes) & -8.36 & -21.06 to 4.34 & 0.191 & 0.82 & 0.25 to 1.38 & 0.005 \\
\hline Insertion time (min) & & - & & 0.04 & 0.02 to 0.05 & $<0.003$
\end{tabular}

$\mathrm{Cl}$ - confidence interval, MRI - magnetic resonance imaging. Bold denotes statistical significance of $p<0.05 .{ }^{*} \beta$ coefficient with $95 \% \mathrm{Cl}$ obtained through multiple linear regression. $\beta$ represents the change in insertion time (in minutes) or total procedural time (in hours) with the variables displayed 




Fig. 3. Box-whisker plots showing total procedural time by period of insertion and MRI-based planning

In the present study, we found that the duration of applicator insertion was significantly reduced by $27 \%$ (about $10 \mathrm{~min}$ ) in the period of 2019-2020 (mean, $27.8 \mathrm{~min}$ ), compared to the earlier adoption period of 2017-2018 (mean, $38.1 \mathrm{~min}$ ). This observed correlation can be rationalized easily and most likely attributed to the initial phase of familiarization with the procedure. Similar trend of $30 \%$ reduction (about 1.6 hours) in the mean total procedural time was observed in the same period (Figure 3), in part due to reduction in the initial learning curve but largely due to the shift from OT to the insertion suite, which has significantly reduced patients' waiting and transfer time.

Although application of MRI in brachytherapy treatment planning for cervical cancer is desired, its widespread utilization is hampered by longer procedural time, limited availability of MRI, and expensive cost, especially in the current climate of sensitivity to responsible resource prioritization [29-31]. In our study, it is not surprising to discover that the addition of MRIbased planning over $\mathrm{CT}$ increased the total procedural time significantly (5.9 hours vs. 4.1 hours, $p<0.003$ ) (Figure 3). Like many centers in the world, we do not have a dedicated MRI scanner in our department, and it is logistically challenging to obtain an MRI scan at the desired time slot during every brachytherapy treatment. Therefore, several groups have published practical recommendations for CT-based brachytherapy. There is ongoing effort by the European Group of Curietherapie-European Society for Therapeutic Radiology and Oncology (GEC-ESTRO) network to shed some light on this aspect, and work is underway to introduce guidelines for contouring of targets and organs at risk based on CT imaging [32-35].

Our study has several strengths and limitations. This was a study with relatively large sample, where all the insertions were performed by a single radiation oncologist, therefore limiting technical biases as much as possible. To our knowledge, this is the only study to include three different modern hybrid applicators (Venezia, Utrecht, and interstitial ring) that are commercially available. First, we acknowledge that it is difficult to extrapolate from a single-center and single-operator experience, and thus, the generalizability of our findings is limit- ed. In addition, our sedation approach may not be replicated in institutions with different training programs or brachytherapy infrastructure. In our department, all brachytherapy practitioners, medical officers, and specialist trainees providing sedation must have received training accredited by our institution before undertaking independent practice. They must also be trained to manage sedation-related complications, including cardiovascular collapse. Patients should be carefully selected, medically pre-assessed, and informed consent must be obtained. Patients with anxiety or ASA class III or greater are not ideal candidates for this approach, and should be reserved for an anesthetist. A proof of generalizability of our approach would require an external validation by other investigators. Second, our study did not address patient's satisfaction during and after the procedure. Data on patient's scores for pain and discomfort during and immediately after the insertion is lacking. This is largely due to the altered level of consciousness when analgesia and sedative drugs are given during the procedure. Although here, the administration of sedation during the insertions was essentially based on continuous clinical assessment of the patient by the brachytherapist and seditionist, pain scores reported by communicable patients were not documented. However, all the patients reported pain score of 0 to 1 at the time of discharge. Further prospective studies are warranted to evaluate patient's satisfaction profile of sedation.

Despite the various limitations, we consider that this study with a significant number of insertions provides important insights concerning this form of sedation for interstitial brachytherapy, which is so widespread in countries with limited resources. In conclusion, in the proper setting, with trained staff and appropriate set up, outpatient interstitial brachytherapy with non-anesthetist-administered sedation is not only achievable and well tolerated, but also enable the delivery of interstitial brachytherapy in patients who cannot tolerate general or regional anesthesia, in addition to cost and resource containment.

\section{Conclusions}

Outpatient interstitial brachytherapy with non-anesthetist-administered sedation is achievable and can significantly lessen the burden on hospital resources. However, close consultation with an anesthesia department is required for high-risks patients. This method is potentially cost-effective and provides a practical approach in the case of limited access to anesthetist.

\section{Acknowledgements}

We are grateful to Dr Fong Kam Weng for his support. This work was supported in part by the National Cancer Centre Singapore Cancer Fund (grant ID: COMCFYR2017-MAY-SD2).

\section{Disclosure}

The authors report no conflict of interest. 


\section{References}

1. Fokdal L, Sturdza A, Mazeron R et al. Image guided adaptive brachytherapy with combined intracavitary and interstitial technique improves the therapeutic ratio in locally advanced cervical cancer: analysis from the retroEMBRACE study. Radiother Oncol 2016; 120: 434-440.

2. Kamran SC, Manuel MM, Cho LP et al. Comparison of outcomes for MR-guided versus CT-guided high-dose-rate interstitial brachytherapy in women with locally advanced carcinoma of the cervix. Gynecol Oncol 2017; 145: 284-290.

3. Fallon J, Park SJ, Yang L et al. Long term results from a prospective database on high dose rate (HDR) interstitial brachytherapy for primary cervical carcinoma. Gynecol Oncol 2016; S0090-8258(16)31496-2.

4. Agrawal PP, Singhal SS, Neema JP et al. The role of interstitial brachytherapy using template in locally advanced gynecological malignancies. Gynecol Oncol 2005; 99: 169-175.

5. Smith MD, Todd JG, Symonds RP. Analgesia for pelvic brachytherapy. Br J Anaesth 2002; 88: 270-276.

6. Petitt MS, Ackerman RS, Hanna MM et al. Anesthetic and analgesic methods for gynecologic brachytherapy: A meta-analysis and systematic review. Brachytherapy 2020; 19: 328-336.

7. Benrath J, Kozek-Langenecker S, Hupfl M et al. Anaesthesia for brachytherapy $-51 / 2 \mathrm{yr}$ of experience in 1622 procedures. Br J Anaesth 2006 ; 96: 195-200.

8. Williams VM, Kahn JM, Harkenrider MM et al. COVID-19 impact on timing of brachytherapy treatment and strategies for risk mitigation. Brachytherapy 2020; 19: 401-411.

9. Ramirez PT, Chiva L, Eriksson AGZ et al. COVID-19 global pandemic: options for management of gynecologic cancers. Int J Gynecol Cancer 2020; 30: 561-563.

10. American Society of Anesthesiologists: Continuum of depth of sedation: Definition of general anesthesia and levels of sedation/analgesia.

11. Kim TH. Safety and effectiveness of moderate sedation for radiologic non-vascular intervention. Korean J Radiol 2006; 7 125-130.

12. Khader R, Oreadi D, Finkelman $\mathrm{M}$ et al. A prospective randomized controlled trial of two different sedation sequences for third molar removal in adults. J Oral Maxillofac Surg 2015; 73: 224-231.

13. Mamula P, Markowitz JE, Neiswender K et al. Safety of intravenous midazolam and fentanyl for pediatric GI endoscopy: prospective study of 1578 endoscopies. Gastrointest Endosc 2007; 65: 203-210.

14. Bhanabhai H, Samant R, E C et al. Pain assessment during conscious sedation for cervical cancer high-dose-rate brachytherapy. Curr Oncol 2013; 20: e307-310.

15. Guidelines On Safe Sedation Practice for NonAnaesthesiologists in Medical Clinics, including Stand-Alone Ambulatory Surgical Centres and Stand-Alone Endoscopy Suites in Singapore.

16. Practice Guidelines for Sedation and Analgesia by Non-Anesthesiologists. Anesthesiology 2002; 96: 1004-1017.

17. Nomden CN, de Leeuw AAC, Moerland MA et al. Clinical use of the Utrecht applicator for combined intracavitary/ interstitial brachytherapy treatment in locally advanced cervical cancer. Int J Radiat Oncol Biol Phys 2012; 82: 1424-1430.

18. Walter F, Maihofer C, Schuttrumpf L et al. Combined intracavitary and interstitial brachytherapy of cervical cancer using the novel hybrid applicator Venezia: clinical feasibility and initial results. Brachytherapy 2018; 17: 775-781.

19. Smolic M, Sombroek C, Bloemers M et al. Needle use and dosimetric evaluation in cervical cancer brachytherapy using the Utrecht applicator. Radiother Oncol 2018; 126: 411-416.
20. Karlsson L, Thunberg P, With A et al. 3D image-based adapted high-dose-rate brachytherapy in cervical cancer with and without interstitial needles: measurement of applicator shift between imaging and dose delivery. J Contemp Brachytherapy 2017; 9: 52-58.

21. Bhatla N, Berek JS, Cuello Fredes M et al. Revised FIGO staging for carcinoma of the cervix uteri. Int J Gynaecol Obstet 2019; 145: 129-135.

22. American Society of Anesthesiologists. ASA Physical Status Classification System.

23. Froehlich F, Gonvers JJ, Fried M. Conscious sedation, clinically relevant complications and monitoring of endoscopy: results of a nationwide survey in Switzerland. Endoscopy 1994; 26: 231-234.

24. Johnson QL, Borsheski R. Pain management mini-series part III. Procedural sedation for the non-anesthesia provider. Mo Med 2013; 110: 355-360.

25. Leong YH, Tan KHS, Choo BA et al. Novel anesthetic technique for combined intracavitary and interstitial brachytherapy for cervix cancer in an outpatient setting. J Contemp Brachytherapy 2017; 9: 236-241.

26. Dimopoulos JCA, Kirisits C, Petric P et al. The Vienna applicator for combined intracavitary and interstitial brachytherapy of cervical cancer: clinical feasibility and preliminary results. Int J Radiat Oncol Biol Phys 2006; 66: 83-90.

27. Liu ZS, Guo J, Zhao YZ et al. Computed tomography-guided interstitial brachytherapy for locally advanced cervical cancer: introduction of the technique and a comparison of dosimetry with conventional intracavitary brachytherapy. Int J Gynecol Cancer 2017; 27: 768-775.

28. Mayadev J, Qi L, Lentz S et al. Implant time and process efficiency for CT-guided high-dose-rate brachytherapy for cervical cancer. Brachytherapy 2014; 13: 233-239.

29. Trifiletti DM, Libby B, Feuerlein $S$ et al. Implementing MRIbased target delineation for cervical cancer treatment within a rapid workflow environment for image-guided brachytherapy: A practical approach for centers without in-room MRI. Brachytherapy 2015; 14: 905-909.

30. Haie-Meder C, Potter R, Van Limbergen E et al. Recommendations from Gynaecological (GYN) GEC-ESTRO Working Group (I): concepts and terms in 3D image based 3D treatment planning in cervix cancer brachytherapy with emphasis on MRI assessment of GTV and CTV. Radiother Oncol 2005; 74: 235-245.

31. Potter R, Georg P, Dimopoulos JC et al. Clinical outcome of protocol based image (MRI) guided adaptive brachytherapy combined with 3D conformal radiotherapy with or without chemotherapy in patients with locally advanced cervical cancer. Radiother Oncol 2011; 100: 116-123.

32. Viswanathan AN, Dimopoulos J, Kirisits C et al. Computed tomography versus magnetic resonance imaging-based contouring in cervical cancer brachytherapy: results of a prospective trial and preliminary guidelines for standardized contours. Int J Radiat Oncol Biol Phys 2007; 68: 491-498.

33. Ohno T, Wakatsuki M, Toita T et al. Recommendations for high-risk clinical target volume definition with computed tomography for three-dimensional image-guided brachytherapy in cervical cancer patients. J Radiat Res 2017; 58: 341-350.

34. Murakami N, Kasamatsu T, Wakita A et al. CT based three dimensional dose-volume evaluations for high-dose rate intracavitary brachytherapy for cervical cancer. BMC Cancer 2014; 14: 447

35. Nesvacil N, Pötter R, Sturdza A et al. Adaptive image guided brachytherapy for cervical cancer: A combined MRI-/ CT-planning technique with MRI only at first fraction. Radiother Oncol 2013; 107: 75-81. 